\section{Study on swelling behaviour of HANP/PVA composites with adding PLGA for alveolar ridge preservation}

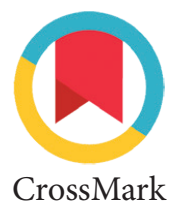

\author{
Feni Istikharoh, ${ }^{1,5 *}$ Hidayat Sujuti, ${ }^{2}$ Edi Mustamsir, ${ }^{3}$ Astika Swastirani ${ }^{4}$
}

${ }^{1}$ Department of Dental Materials, Faculty of Dentistry, Universitas Brawijaya, Malang, Indonesia ${ }^{2}$ Department of Biochemistry, Faculty of Medicine, Universitas Brawijaya, Malang, Indonesia ${ }^{3}$ Department of Orthopaedic, Faculty of Medicine, Universitas Brawijaya, Malang, Indonesia ${ }^{4}$ Department of Oral Medicine, Faculty of Dentistry, Universitas Brawijaya, Malang, Indonesia ${ }^{5}$ Department of Biomedical Science, Faculty of Medicine, Universitas Brawijaya, Malang, Indonesia
*Correspondence to: Feni Istikharoh, Department of Dental Materials, Faculty of Dentistry, Universitas Brawijaya, Malang, Indonesia/Department of Biomedical Science, Faculty of Medicine, Universitas Brawijaya, Malang, Indonesia

feni.istikharoh@ub.ac.id

Received: 28 January 2020

Revised: 20 June 2020

Accepted: 23 March 2021

Available Online: 1 December 2021

\title{
Abstract
}

Objective: The aim of this study was to compare the swelling behaviour of HANP/PVA and HANP/PLGA/PVA composites. Material and Methods: This study was divided into 2 groups, HANP/ PVA and HANP/PLGA/PVA composites which prepared by freeze drying methods. HANP 20\% (w/w) was combined with PLGA 20\% $(\mathrm{w} / \mathrm{w})$ and dissolved in PVA solution at $50^{\circ} \mathrm{C}$. Swelling behaviour was obtained by measured initial weight (Wi) and the weight of composites after immersing in $\mathrm{PBS} \mathrm{pH} 7.4 ; 37^{\circ} \mathrm{C}$ for 7 days (Ws). Statistical analysis based on Independent T-test $(p<0.05)$.

Results: The initial weight (Wi) of HANP/PVA and HANP/PLGA/PVA composites are $467 \pm 4.73$ and $292 \pm 9.52$. The swollen weight (Ws) of HANP/PVA and HANP/PLGA/PVA composites are 897 \pm 26.61 and $860 \pm 32.36$. Furthermore, the water retention of HANP/PVA composite is $92.07 \%(p<0.05)$ and HANP/PLGA/PVA composite is $194.52 \%$ $(p<0.05)$.

Conclusion: The addition of PLGA enhance swelling behaviour of HANP/PVA composites. The strong interaction between HANP and PVA decrease the swelling re. HANP is inert in nature, but the combination of HANP/PLGA/PVA can be a good substitute for alveolar preservation.

Keywords: Swelling behaviour, HANP, PVA, PLGA, Alveolar ridge preservation

Cite this Article: Istikharoh F, Sujuti H, Mustamsir E, Swastirani A. 2021. Study on swelling behaviour of HANP/PVA composites with adding PLGA for alveolar ridge preservation. Journal of Dentomaxillofacial Science 6(3): 197-199. D0l: 10.15562/jdmfs.v6i3.998

\section{Introduction}

Alveolar bone resorbtion can be occured in several physiological and pathological process, e.g. post tooth extraction, periodontitis, etc. ${ }^{1}$ The incident of bone resoption made worst if the patients have systemic disease e.g., diabetes mellitus. ${ }^{1,2}$ When alveolar bone resoption is occured, it cannot regenerate in normal physiological processes, so requires other intervention such as bone graft. Bone resorption after tooth extraction treatment is physiological and progressive process, especially in the first 6 month. ${ }_{3,4}$ Futhermore, alveolar bone resorption ma compromise dental implant, removable and fix denture treatment with risk destroyed anatomical structure. $^{2-4}$ Therefore, alveolar ridge preservation is one methods to avoid aggresive bone loss after tooth extraction treatment. ${ }^{5}$

Biocomposite based on polymers and ceramics have been developed for biomedical application, especially for bone repair and reconstruction. ${ }^{6}$ Several polymers which widely used for this purpose are Polylactic co-glycolic acid (PLGA) and Poly Vinyl Alcohol (PVA) because of their biocompatibility and biodegradability properties. PLGA is one of the best biodegradable materials, drug carrier and can be degrade to non-toxic products. ${ }^{7}$ PVA has great biocompatibility, physicochemical characteristic and has been used for biomedical application. PVA has better mechanical stability than other polymers which has been demonstrated in previous studies. ${ }^{8}$

Hydroxyapatite Nanoparticles (HANP) is natural bioceramic extracted from many sources, e.g. scales of fish. HANP shows dimentional stability, improve composite density, and fracture toughness. ${ }^{9}$ Furthermore, HANP has better osteoconductivity, biocompatibility, and biodegradability if combine with other materials to be composites. As in study, HANP/PVA composites has slight microporosity and it should combines with another material to improve the microstructure of HANP/PVA composites. ${ }^{10}$ The aim of our study was to evaluate the effect of adding PLGA on swelling behaviour of HANP/PVA composites, measuring for 7 days.

\section{Material and Methods}

HANP ( 60 nmBATAN, Indonesia), PVA (fully hydrolysed, Mw 73.000, Merck, USA), PLGA (50:50, acid terminated, PolysciTech, USA), PBS (Merck, USA).

\section{Preparation HANP/PVA/PLGA composites}

At first, PVA was dissolved in distilled water at $50^{\circ} \mathrm{C}$ for 2 hours by magnetic stirrer. In other tube. HANP 20\% (w/w) was added in PVA solution at room temperature, then PLGA 20\% (w/w) was added and stirred until the solution homogenous. The PVA/HANP/ PLGA solution was distributed into 48 well plate. The well plates were placed within a deep freezer at $-80^{\circ} \mathrm{C}$ for $24 \mathrm{~h}$. 


\section{Swelling behaviour}

In this study, swelling behaviour was investigated according a method describes earlier. All of the composites were fully immersed in PBS solution ( $\mathrm{pH}$ 7.4) for seven days. The swelling percentage of the samples were calculated by the equation:

Water retention $(\%)=\frac{\mathrm{Ws}-\mathrm{Wi}}{\mathrm{Wi}} \times 100$

Where Ws is the weight of sampels after swelling and $\mathrm{Wi}$ is an initial weight conditions $(\mathrm{n}=3)$. The values were expressed as percentage.

\section{Swelling Behaviour}

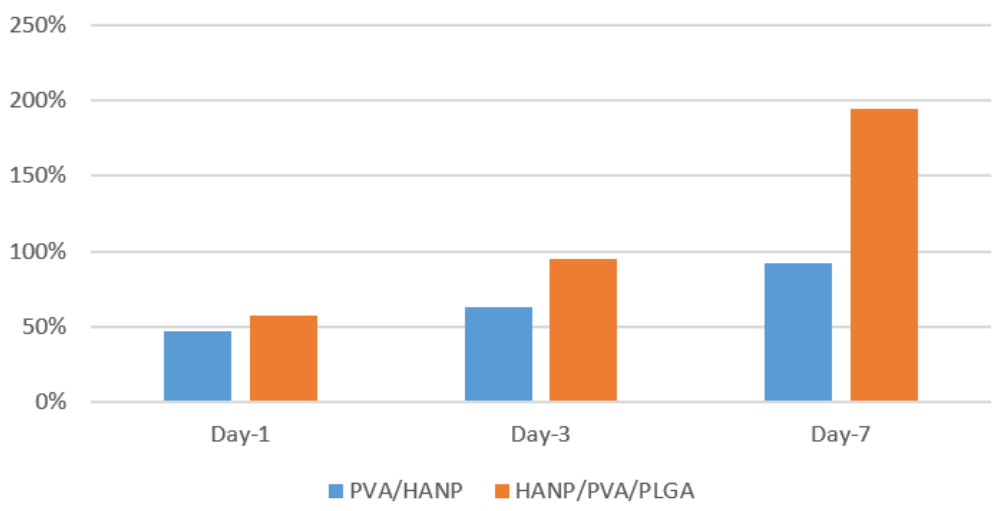

\section{Figure 1 Swelling HANP/PVA and HANP/PVA/PLGA after 7 days}

\section{Results}

Swelling studies indicated that the HANP/ PVA composites which is added PLGA absorb water more than that of initial weight after 7 days. There is slight difference between adding PLGA or not in after $24 \mathrm{~h}$ in PBS. The HANP/ PVA/ PLGA composites raises gradually the swelling percentage after soaked $1 \mathrm{~d}, 3 \mathrm{~d}$ and $7 \mathrm{~d}$ in PBS. The HANP/PVA composites without PLGA also raises until day-7 but not as great as HANP/ PVA/ PLGA composites figure 1.

\section{Discussion}

Besides structural stability, the swelling behaviour is one of the critical for their practical use in tissue engineering especially for alveolar ridge presevation. ${ }^{11,12}$ Swelling of composites allows absorption of body fluid and transfer of cell nutrients and metabolites inside the scaffold. ${ }^{12}$ Swelling behaviour is depending with the porosity and the natural of materials which contained in the composites. ${ }^{12}$ To assess the swelling behavior of the composites or scaffolds, the samples should be soaked in culture media (DMEM, AMEM), PBS or SBF for several days up to three weeks. ${ }^{13}$ In our study, all of the composites were soaked in PBS for seven days and there is slight apparent size/shape change for all the scaffold. HANP enhances apatite like structur formation by supporting chemical interaction on formability of HANP/PVA composites. ${ }^{8}$ The freeze dried composite in this study showed high swelling ability. This observation shows that addition PLGA has a beneficial effect. The incorporation of PLGA in composites may increase the swelling capability because PLGA can increase the porosity of scaffold. With adding PLGA, the swelling increase which is attributed to hydrophilic functional groups. ${ }^{7,14}$ Furthermore, PLGA may increase the swelling percentage may be credited to the disturbace of hydroxyl group of HANP and PVA molecules. Swelling behaviour of composites or scaffolds is helpful for stable implantation and helps to fill a specific tissue defect..$^{15}$ Furthermore, the swelling of HANP composites in an aquous environment is an important property because swelling behaviour increase porosity and surface area to support cell attachment and tissue regenation, thus maximizing the internal surface area of composites for cell infusion and attachment. ${ }^{8,15}$ Swelling is desirable and the resultant increase in pore size facilitates cell attachment and growth. The swelling behaviour was evaluate quantitatively which is defined as the weight difference between wet and dry states of a sample divided by the dry weight of the samples. This ratio reveals how materials absorb aquous media with respect to its dry state.

\section{Conclusion}

The addition of PLGA increase the swelling behaviour of the composites. HANP is inert in nature, so the combination of HANP/PVA/PLGA can be a good substitute for tissue engineering. Therefore, still need further research to be conducted for optimizing the composites. Swelling in physiological must be controlled cause it may weakening and rapid.

\section{Akcnowledgment}

Thank you for the support to Faculty Dentistry Universitas Brawijaya.

\section{Conflict of Interest}

The authors report no conflict of interest. 


\section{References}

1. Baniasadi B, Evrard L. Alveolar ridge preservation after tooth extraction with dfdba and platelet concentrates: A radiographic retrospective study. Dent J 2017;11: 99-108.

2. Levengood SL, Zhang M. Chitosan-based scaffolds for bone tissue engineering. J Mater Chem B Mater Biol Med 2014;2: 3161-3184.

3. Sheikh Z, Najeeb S, Khurshid Z, et al. Biodegradable Materials for Bone Repair and Tissue Engineering Application. Mater 2015;8: 5744-5794.

4. Hansson S, Halldin A. Alveolar ridge resorption after tooth extraction: A consequence of fundamental principle of bone physiology. J Dent Biomech 2012;3: 1-6.

5. Hong CE, Lee JY, Joo JY. Prediction the alveolar bone level after the extraction of maxillary anterior teeth with severe periodontitis. J Periodon Imp Sci 2015;45: 216-222.

6. Loh QH, Choong C. Three-Dimensional scaffold scaffolds for tissue engineering applications: Role of porosity and pore size. Tiss Eng Part B 2013;19: 485-502.

7. Karthik V, Pabi SK, Chowdhury SKR. Development of hydroxyapatite/polyvinyl alcohol bionanocomposite for prothesis implants. Mater Sci Eng 2018;314: 1-6.

8. Kumar P, Dehiya BS, Sindhu A. Ibuprofen-Loaded CTS/ nHA/nBG Scaffolds for the applications of hard tissue engineering. Iran Biomed J 2019;23: 190-199.

9. Dewi AH, Ana ID. The use of hydroxyapatite bone substitute grafting for alveolar ridge preservation, sinus augmentation, and periodontal bone defect: A systematic review. Heliyon 2018;4: 1-10.
10. Kumar P. Nano-TiO2 doped chitosan scaffold for the bone tissue engineering applications. Int J Biomater 2018;7: 1-8.

11. Maji K, Dasgupta S, Pramanik K, et al. Preparation and evaluation of gelatin-chitosan-nanobioglass $3 \mathrm{~d}$ porous scaffold for bone tissue engineering. Int J Biomater 2016;2016: 14.

12. Jana S, Florczyk SJ, Leung M, et al. High-strength pristine porous chitosan scaffolds for tissue engineering. J Mater Chem 2012;22: 6291.

13. Kapoor DN, Bhatia A, Kaur R, et al. PLGA: A unique polymer for drug delivery. Ther Deliv 2015;6: 41-58.

14. Balgova Z, Palou M, Wasserbauer J, et al. Synthesis of poly (vinyl alcohol) - hydroxyapatite composites and characterization of their bioactivity. Cent Eur J Chem 2013;9: 1401-1411.

15. Ashraf AA, Zebarjad SM, Hadianfard MJ. The cross-linked polyvinyl alcohol/hydroxyapatite nanocomposite foam. J Mater Res Technol 2019;8: 3149-3157.

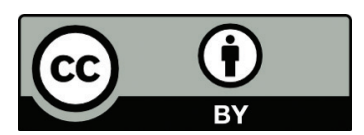

This work is licensed under a Creative Commons Attribution 